\title{
Danazol regulates the functions of normal human endometrial stromal cell subpopulations by modifying endometrial cytokine networks
}

\author{
TETSUJI TANAKA \\ Department of Obstetrics and Gynecology, Wakayama Medical University, 811-1 Kimi-idera, Wakayama 641-0012, Japan
}

Received September 23, 2008; Accepted November 24, 2008

DOI: 10.3892/ijmm_00000147

\begin{abstract}
Local danazol therapy can improve endometriotic signs and symptoms without causing any menstrual disorders. As a consequence, certain direct actions of danazol on endometriotic tissues have been proposed, but the mechanisms of these actions have not been clarified. In the present study, the direct effects of danazol on normal human endometrial stromal cells (ESCs) were examined using in vitro decidualization assays. Danazol did not affect the viable cell numbers of unstimulated ESCs or 8Br-cAMP-stimulated decidualized ESCs, but significantly enhanced the viable cell numbers of 8-Br-cAMP-stimulated ESCs during decidualization in a dose-dependent manner. Danazol had no effect on PRL secretion by 8 -Br-cAMP-stimulated decidualized ESCs. Danazol, as well as progesterone and medroxyprogesterone acetate (MPA), induced ESC decidualization. Danazol synergistically enhanced the differentiation process of $8-\mathrm{Br}$ cAMP-stimulated ESCs during decidualization. Although progesterone and MPA increased G-CSF and IL-8 secretion by ESCs in similar manner to 8-Br-cAMP, danazol had no such effects. Moreover, remarkable increases in G-CSF and IL-8 secretions by 8 -Br-cAMP-stimulated ESCs during decidualization were completely inhibited by cotreatment with danazol. These results indicate that danazol has specific pharmacological effects on ESCs, rather than progesteronelike effects or similar effects to those reported for endometrial cytokines. According to the results, normal human ESCs can be classified into at least four functional subpopulations. Therefore, under certain circumstances, danazol has similar or opposite effects on ESCs to certain endometrial cytokines, suggesting that it regulates functional cellular subpopulation ratios of normal human ESCs by modifying the endometrial cytokine network in endometrial stromal tissues.
\end{abstract}

Correspondence to: Dr Tetsuji Tanaka, Department of Obstetrics and Gynecology, Wakayama Medical University, 811-1 Kimi-idera, Wakayama 641-0012, Japan

E-mail: obgywmu@wakayama-med.ac.jp

Key words: danazol, endometriosis, stromal cell, decidualization, granulocyte-colony stimulating factor, interleukin- 8

\section{Introduction}

Danazol, a progesterone-like antiendometriosis medicine $(1,2)$, has been applied clinically for endometriotic and adenomyotic patients for several decades throughout the world, although its mechanisms of action were unclear. The standard dose of oral danazol for endometriotic patients is $400-800 \mathrm{mg} /$ day, which inhibits ovarian estrogen production and the luteinizing hormone surge from the pituitary gland and causes dysovulation and amenorrhea (3-12). Thus, orally administered danazol inhibits endogenous estrogen production and acts as a progesterone-like steroid hormone. The abovedescribed effects are widely believed to be the main mechanisms by which oral danazol therapy prevents endometriosis, an estrogen-dependent proliferative disease with extrauterine endometrium-like tissue formation. When endometriotic patients are administered danazol at $200-800 \mathrm{mg} / \mathrm{day}$, endometrial atrophy occurs (9). However, local administration of low-dose danazol via vaginal danazol suppositories (13-16), cervical injections of danazol suspensions (17) or use of intrauterine danazol rings $(13,18-19)$ can improve endometriotic signs and symptoms without causing any menstrual disorders. Local danazol therapy does not inhibit either endogenous estrogen production or ovulation, in contrast to the effects of oral danazol therapy $(13,14,16)$. From these clinical data, certain direct actions of danazol on endometriotic tissues have been proposed, but the mechanisms of these actions have not been clarified. Understanding how local danazol therapy is pharmacologically effective for endometriosis may provide clues toward elucidating the causes or pathogenesis of endometriosis.

There have been several studies of direct actions of danazol on endometrial epithelial cells (20-24). In these studies, the direct effects of danazol were examined using the highlydifferentiated endometrial adenocarcinoma cell line Ishikawa (20), surgically excised endometrial carcinoma cells (21) or normal human endometrial cells (22-24). The respective investigators all concluded that danazol works directly on the cells by inhibiting their proliferation. However, these conclusions are not fully convincing because no complications such as amenorrhea or endometrial ulcers due to endometrial atrophy and no vaginal ulcer lesions or destructive changes in the uterine cervix have ever been reported during local danazol therapy with vaginal danazol suppositories (13-16) or cervical injections of danazol suspensions (17). To clarify this clinical 
discrepancy, we previously carried out in vitro culture experiments using two kinds of human endometrial adenocarcinoma cell lines and purified normal human endometrial stromal cells (ESCs) (25). Although previous in vitro studies reported that danazol suppressed both endometrial epithelial cell growth (20-23) and ESC growth $(24,26)$, we found that thoroughly dissolved danazol exhibited no growth suppression of endometrial epithelial cells or ESCs (25) at a concentration of $500 \mathrm{ng} / \mathrm{ml}$, which is $\sim 10$-fold higher than the serum danazol levels in patients during oral danazol therapy (11). Serum and peritoneal fluid danazol concentrations were reported to be $<5 \mathrm{ng} / \mathrm{ml}$ in endometriotic patients who received danazol vaginal suppositories and exhibited improvement in their endometriotic symptoms without any apparent disturbances of endogenous estrogen production or ovulation $(13,14,16)$.

Regarding endometrial epithelial cells, we recently demonstrated that thoroughly dissolved danazol enhances Fasmediated apoptotic signals but not anticancer drug-induced apoptotic signals in the human endometrial epithelial cell line HHUA (27). Endometrial epithelial cell growth and viability can be regulated in vivo by ESCs in paracrine manner. If certain functions of ESCs are directly inhibited by danazol as previously reported $(24,26)$, danazol-treated ESCs may inhibit endometrial epithelial cell proliferation. However, we found that thoroughly dissolved danazol had no effects on the proliferation of endometrial epithelial cells or ESCs (25). In the present study, we examined the direct effects of danazol on ESCs using in vitro decidualization assays (28).

\section{Materials and methods}

Preparation of danazol solution. Danazol powder was kindly provided by Tokyo-Tanabe Pharmaceutical Co. Ltd. (Tokyo, Japan). A danazol stock solution $(10 \mathrm{mg} / \mathrm{ml}$ danazol in $99.5 \%$ ethanol) was prepared as described previously (25). Danazol is less soluble in aqueous solutions than in solvents. When the danazol stock solution was diluted directly with culture media and added to cell cultures, the culture media became white and turbid, and insoluble danazol crystals were detected in the cultures. Therefore, to achieve thorough dissolution of danazol in culture media, the danazol stock solution was first diluted with ethanol to create a solution of $100 \mu \mathrm{g} / \mathrm{ml}$ danazol in ethanol. Next, this solution was diluted with culture medium to create a solution of $5 \mu \mathrm{g} / \mathrm{ml}$ danazol in $5 \%(\mathrm{v} / \mathrm{v})$ ethanol, and then diluted stepwise with culture medium containing $5 \%(\mathrm{v} / \mathrm{v})$ ethanol. A one-ninth volume of the stepwise-diluted solution was added to each cell culture so that all the cultures contained $0.5 \%(\mathrm{v} / \mathrm{v})$ ethanol. A final concentration of $0.5 \%$ $(\mathrm{v} / \mathrm{v})$ ethanol was also added to control cell cultures. Using this dilution method, insoluble danazol crystals and white turbidity were not observed in cell cultures.

Isolation and culture of human ESCs. In the present study, we obtained normal ESCs from non-endometriotic patients with normal menstrual cycles. Briefly, non-endometriotic patients with early uterine cervical cancers gave informed consent for isolation of normal ESCs from their surgically excised uteri. The endometrial tissue samples were cut into small fragments with scissors, incubated in medium containing
$0.1 \%(\mathrm{w} / \mathrm{v})$ collagenase (Wako Chemicals, Tokyo, Japan) for $30 \mathrm{~min}$ at $37^{\circ} \mathrm{C}$ and filtered twice through $40-\mu \mathrm{m}$ nylon mesh to remove epithelial cells. The isolated ESCs were cultured in OPTI-MEM (Gibco-BRL, Gaithersburg, MD, USA), 5\% fetal calf serum (FCS) (Lot no. SFB30-901; Equitech-Bio Inc., Ingram, TX, USA), penicillin (PC) (100 U/ml), streptomycin (SM) $(100 \mu \mathrm{g} / \mathrm{ml})$ and $17 ß$-estradiol (E2) (100 nM; Sigma, St. Louis, MO, USA). Non-adherent cells were removed by several intensive washes over 2 weeks. More than $99.5 \%$ of the residual adherent cells were confirmed to be vimentinpositive ESCs (data not shown) and used for in vitro differentiation experiments. Differentiation activity was evaluated by measuring prolactin (PRL), granulocyte-colony stimulating factor (G-CSF) and interleukin (IL)-8 secretion by stimulated ESCs.

Viable cell number assay. Viable cell numbers were assayed using an XTT Cell proliferation assay kit (BoehringerMannheim, Mannheim, Germany), which quantified enzyme activity in viable cells based on the absorbance at $450 \mathrm{~nm}$. The effects of danazol and 8-bromo-cyclic adenosine 3', 5'monophosphate (8-Br-cAMP; Sigma) on the cell viability of ESCs were assayed as described below. ESCs were detached with $0.25 \%$ trypsin/ $1 \mathrm{mM}$ EDTA (Gibco-BRL) and cultured to confluence in estradiol-containing medium in 96-well culture plates (2,500 cells/well). On day 1, $0.5 \mathrm{mM} 8$-Br-cAMP and various concentrations of danazol were added to the media. The culture media were exchanged for fresh media on days 4,7 and 10, and the viable cells were counted on day 14 . To examine the effects of danazol on decidualized cell viability, ESCs were cultured with $0.5 \mathrm{mM} \mathrm{8-Br-cAMP}$ without danazol for 10 days, during which time the culture media were exchanged for fresh media on days 4, 7 and 10 . On day 10 , various concentrations of danazol were added to the cultures without 8-Br-cAMP, and the viable cell numbers were assayed on day 14. All experiments were performed 3 times to verify the results. The data were expressed as the mean \pm SEM and analyzed by Student's t-test $(n=6)$ and oneway ANOVA.

In vitro decidualization activity assay. ESCs from three patients were subjected to in vitro decidualization assays (28) that were repeated at least 3 times. Briefly, ESCs were detached with $0.25 \%$ trypsin/ $1 \mathrm{mM}$ EDTA and cultured with OPTI-MEM/FCS/PC/SM/E2 to confluence in 96-well culture plates. To induce in vitro decidualization, the cells were cultured with $0.5 \mathrm{mM}$ 8-Br-cAMP for 10-14 days. The optimal concentration and stimulation period of 8-Br-cAMP were determined in a previous study (29). Decidualization activity was evaluated by PRL secretion by the stimulated ESCs. To examine the dose-dependent effects of danazol on decidualization, various concentrations of danazol were added to the medium during or after decidualization. The culture media were exchanged for fresh media on days 4, 7 and 10, and the supernatants were stored at $-20^{\circ} \mathrm{C}$ until analysis for their PRL, G-CSF and IL- 8 concentrations. The data reported in this study represent the accumulated PRL, G-CSF and IL-8 amounts in the culture supernatants during the last 4 days of culture. All the experiments were performed 3 times for one of the three ESC preparations to verify the results. 
A

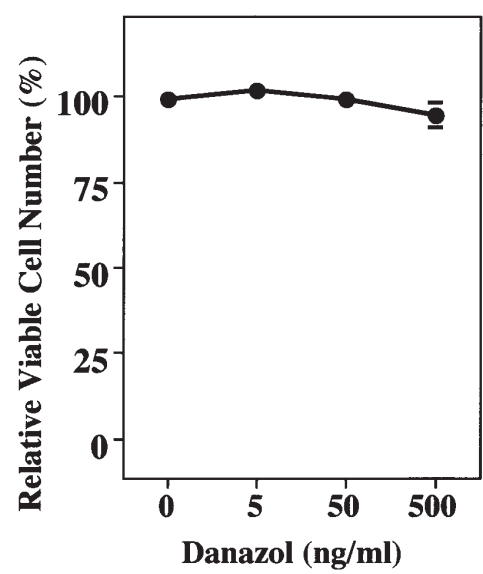

B

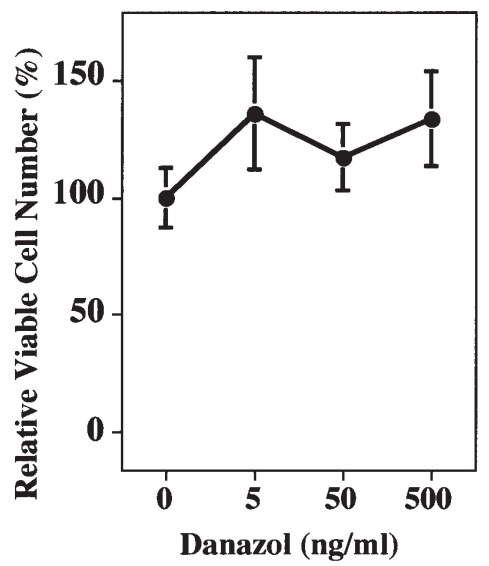

C

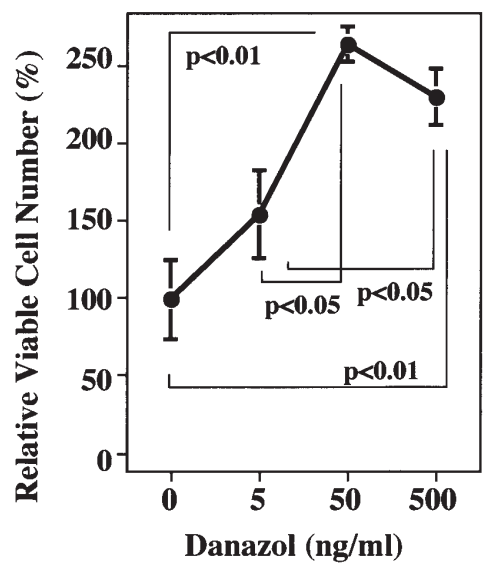

Figure 1. Effects of danazol on viable cell numbers of unstimulated, decidualizing and decidualized ESCs. (A) Unstimulated ESCs. The ESCs were treated with danazol. (B) Decidualized ESCs. 8-Br-cAMP-stimulated ESCs comprising maximally decidualized cells were treated with danazol without 8-Br-cAMP. (C) Decidualizing ESCs. The ESCs were simultaneously treated with danazol and 8-Br-cAMP. The cells are in the process of decidualization.

To examine the effects of progesterone (Sigma) and medroxyprogesterone acetate (MPA; Sigma), ESCs were cultured with progesterone or MPA for 14 days with exchange of the culture media for fresh media on days 4,7 and 10 . The culture supernatants for the last 4 days of culture were stored at $-20^{\circ} \mathrm{C}$ until analysis for their PRL, G-CSF and IL-8 concentrations carried out in duplicate.

Enzyme immunoassays of PRL, G-CSF and IL-8 concentrations in culture supernatants. The PRL concentrations in culture supernatants were assayed with an enzyme immunoassay instrument (SR-1; Biochem Immunosystems, Rome, Italy). The G-CSF and IL- 8 concentrations in culture supernatants were assayed by enzyme-linked immunosorbent assay kits for G-CSF (IBL Co., Maebashi, Japan) and IL-8 (Genzyme Techne, Minneapolis, MN, USA). All experiments were performed 3 times. The data $(n=4)$ were analyzed by one-way ANOVA.

\section{Results}

Direct effects of danazol on viable cell numbers of unstimulated, decidualizing and decidualized ESCs. The direct effects of danazol on the viable cell numbers of unstimulated, decidualizing and decidualized ESCs were examined using a previously reported in vitro decidualization assay (28). Changes in the viable cell numbers were evaluated when the mean viable cell numbers without danazol were determined as a viable cell ratio of $1(100 \%)$. As shown in Fig. 1A, danazol alone had no effect on the proliferation of unstimulated ESCs. Stimulation with $500 \mathrm{ng} / \mathrm{ml}$ danazol, which is 10-fold higher than the serum danazol concentrations during usual oral danazol therapy, did not decrease the viable cell numbers.

ESCs stimulated with 8-Br-cAMP differentiate into PRLsecreting decidualized cells and die within 1-2 weeks after activation (28). As shown in Fig. 1B, danazol did not affect the viable cell numbers of 8 -Br-cAMP-stimulated decidualized cells. However, As shown in Fig. 1C, danazol significantly enhanced the viable cell numbers of 8-Br-cAMP-stimulated ESCs during decidualization, indicating that it strongly inhibits 8-Br-cAMP-activated cell death and may enhance the viability of differentiating cells during the process of decidualization.

Effects of danazol on ESC differentiation into PRL-secreting cells. Under stimulation with 8-Br-cAMP, ESCs differentiate into decidualized cells that produce PRL (28). Therefore, we examined the effects of danazol on 8-Br-cAMP-stimulated decidualization of ESCs. The decidualization activity was evaluated by measuring the PRL concentrations in culture supernatants relative to the viable cell numbers $(\mathrm{ng} / \mathrm{ml} /$ viable cell ratio) when the mean viable cell numbers without danazol were determined as a viable cell ratio of $1(100 \%)$. Although unstimulated ESCs secreted very little PRL, danazol dosedependently enhanced their PRL secretion (Fig. 2A). However, danazol did not affect PRL secretion by 8-Br-cAMP-stimulated decidualized ESCs (Fig. 2B). Interestingly, danazol dosedependently increased PRL secretion by 8-Br-cAMPstimulated ESCs during decidualization (Fig. 2C). This means that the cell ratios of PRL-secreting cells increased among 8-Br-cAMP-stimulated ESCs during decidualization, indicating that danazol increased not only the viable cell numbers but also the decidualized cell numbers. In other words, danazol remarkably enhances the decidualization process of ESCs stimulated with 8-Br-cAMP.

Effects of danazol on G-CSF and IL-8 secretion by 8-BrcAMP-stimulated ESCs. There have been studies that 8-BrcAMP induces ESC activation into G-CSF-secreting (30) and IL-8-secreting (31) cells. Therefore, the effects of danazol on G-CSF and IL-8 secretion by 8-Br-cAMP-stimulated decidualized cells were examined. The activation activity was evaluated by measuring the G-CSF and IL-8 concentrations in culture supernatants relative to the viable cell numbers ( $\mathrm{pg} / \mathrm{ml} /$ viable cell ratio) when the mean viable cell numbers without danazol were determined as a viable cell ratio of 1 

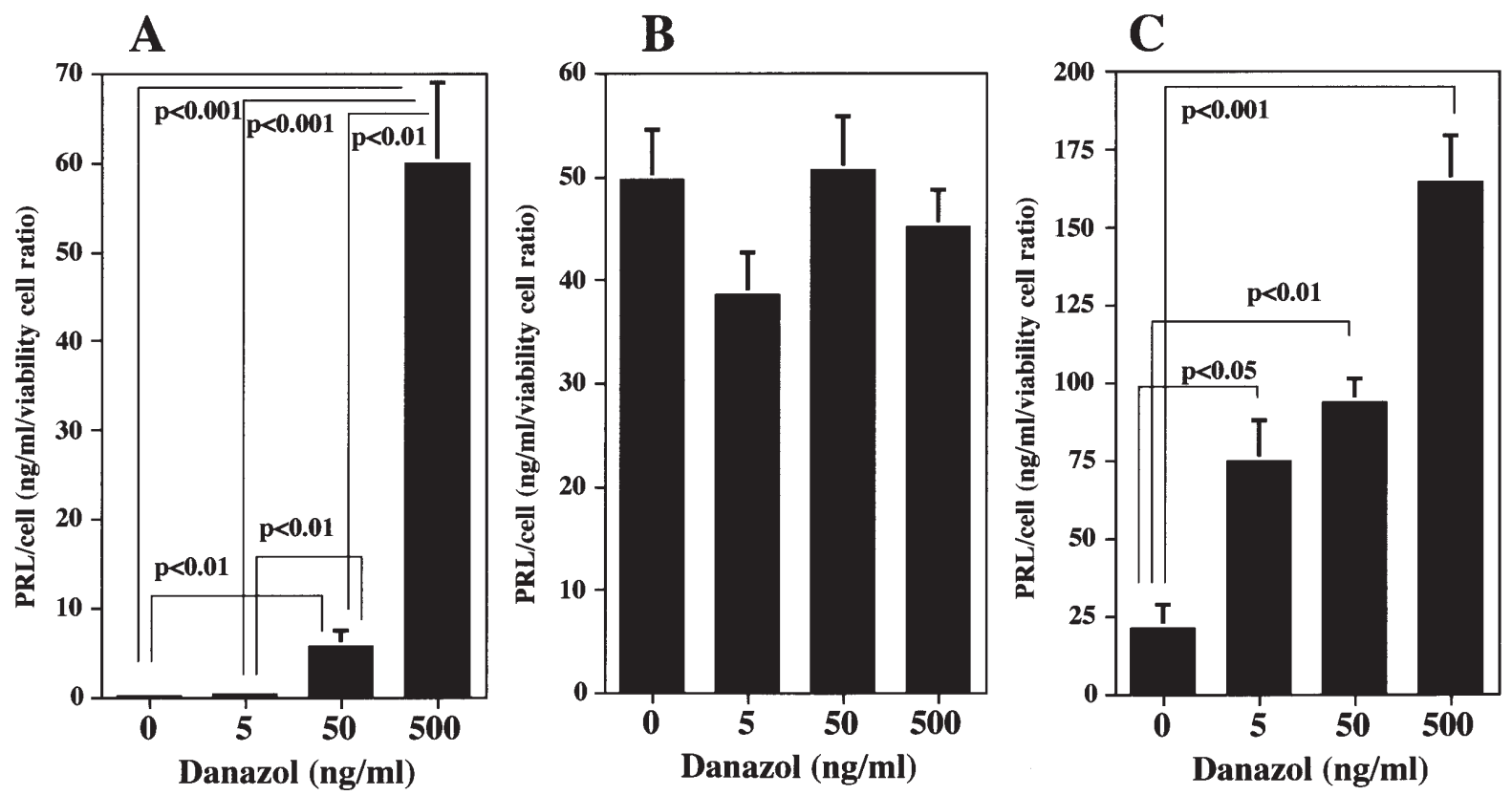

Figure 2. Effects of danazol on ESC differentiation into PRL-secreting cells. (A) Unstimulated ESCs. The ESCs were treated with danazol. (B) Decidualized ESCs. 8-Br-cAMP-stimulated ESCs comprising maximally decidualized cells were treated with danazol without 8-Br-cAMP. (C) Decidualizing ESCs. The ESCs were simultaneously treated with danazol and 8-Br-cAMP. The cells are in the process of decidualization.
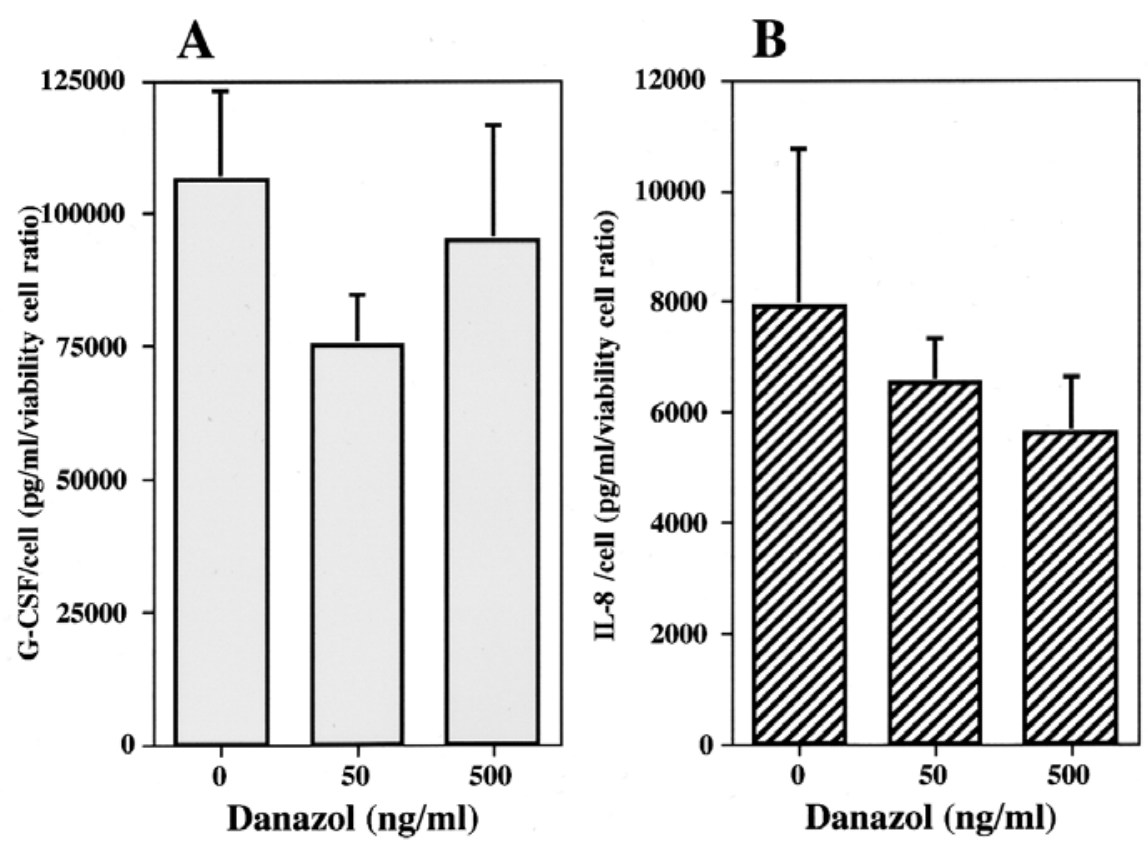

Figure 3. Effects of danazol on G-CSF and IL-8 secretion by 8-Br-cAMP-stimulated ESCs. 8-Br-cAMP-stimulated ESCs comprising maximally decidualized cells were treated with danazol without 8-Br-cAMP. The G-CSF and IL-8 concentrations in their culture supernatants were assayed. (A) G-CSF secretion; (B) IL-8 secretion. Danazol has no effects on G-CSF or IL-8 secretion by 8-Br-cAMP-stimulated decidualized ESCs.

(100\%). As shown in Fig. 3, danazol had no effects on G-CSF and IL-8 secretion by 8 -Br-cAMP-stimulated decidualized ESCs.

Effects of danazol on ESC activation into G-CSF- and IL-8secreting cells. The effects of danazol on ESC activation into G-CSF- and IL-8-secreting cells were examined. Unstimulated ESCs secreted small amounts of G-CSF and IL-8. In the absence of 8Br-cAMP stimulation, danazol treatment did not affect either G-CSF or IL-8 secretion by ESCs (Fig. 4). Although 8-Br-cAMP stimulation induced significant increases in G-CSF and IL- 8 secretion by ESCs, addition of danazol remarkably decreased the G-CSF and IL-8 secretion by 8-Br-cAMP-stimulated ESCs to their basal secretion levels by unstimulated ESCs. These results suggest that danazol does not inhibit G-CSF and IL-8 secretion by undifferentiated 
A

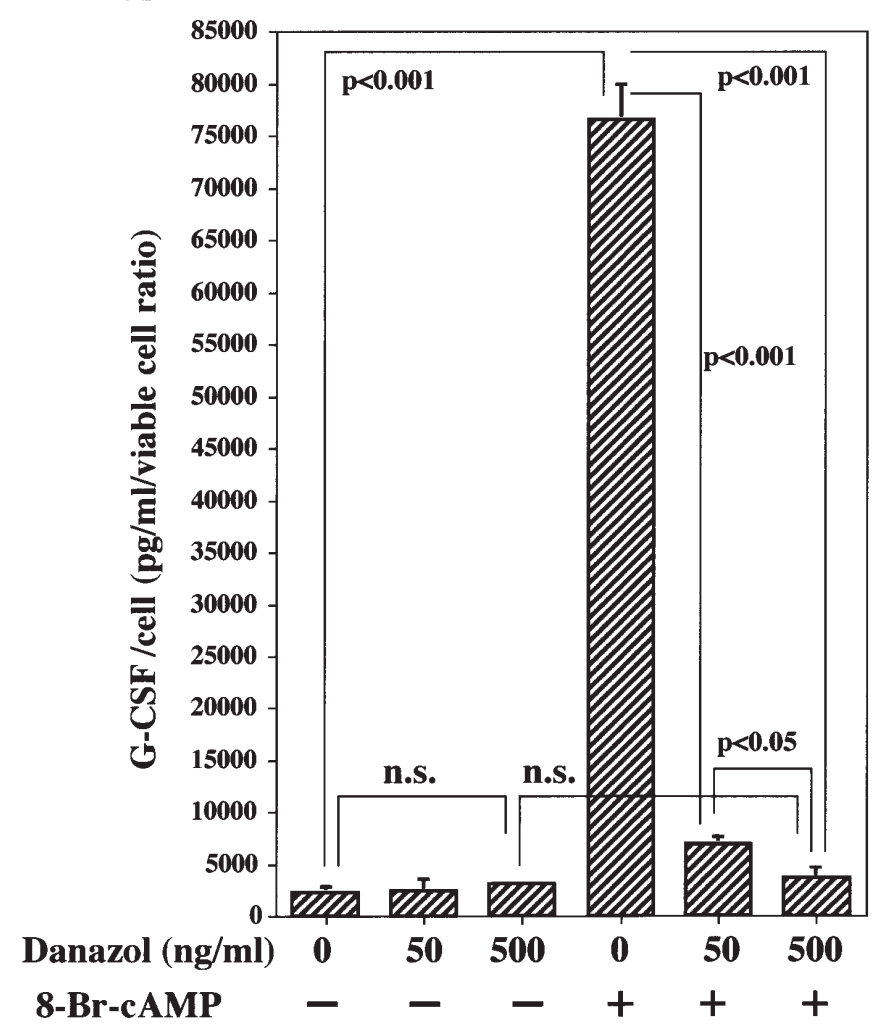

B

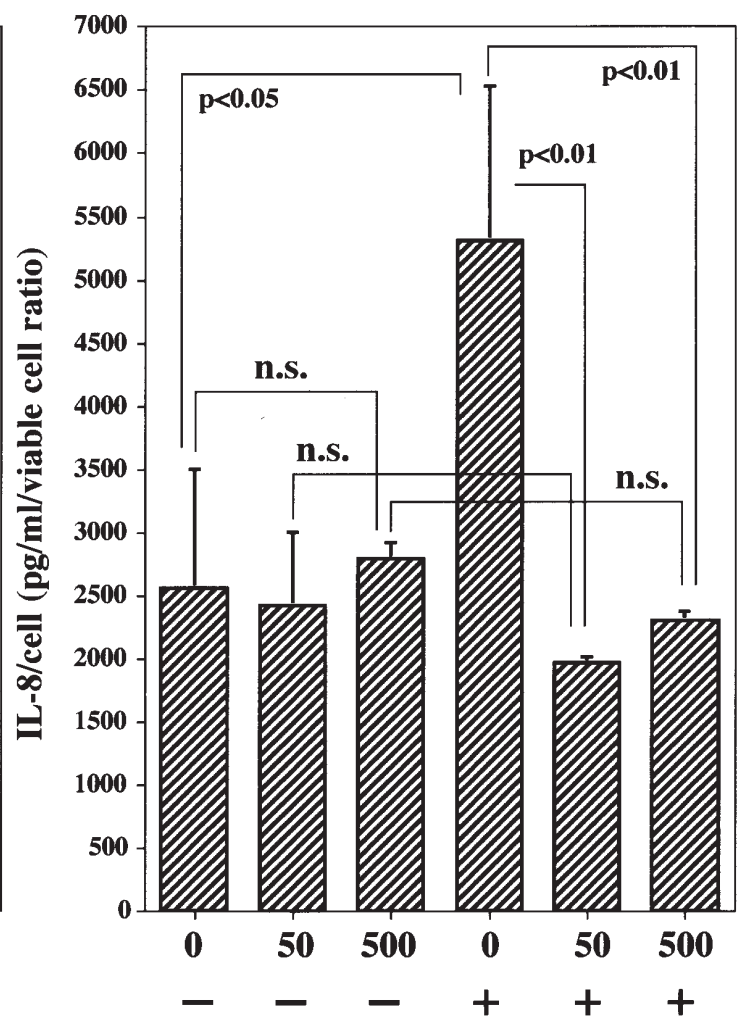

Figure 4. Effects of danazol on ESC activation into G-CSF- and IL-8-secreting cells. (A) G-CSF secretion; (B) IL-8 secretion; n.s., not significant.

ESCs and almost completely inhibits the 8-Br-cAMP-induced ESC activation process into G-CSF- and IL-8-secreting cells.

Effects of progesterone and MPA on PRL, G-CSF and IL-8 secretion by ESCs. Danazol is reported to have progesteronelike actions $(1,2)$. Progesterone is believed to be the most important physiological inducer of ESC decidualization. The observation that danazol enhanced the differentiation of ESCs into PRL-secreting cells (Fig. 2A) may reflect a progesterone-like action of danazol. As shown in Fig. 4, danazol almost completely inhibited the 8-Br-cAMP-induced ESC activation process into G-CSF- and IL-8-secreting cells. Therefore, to examine whether the effects of danazol on G-CSF and IL-8 secretion by 8 -Br-cAMP-stimulated ESCs during decidualization were progesterone-like actions, the same ESCs assayed above were cultured with progesterone and MPA. As shown in Fig. 5A and D, progesterone and MPA induced the differentiation of ESCs into PRL-secreting cells. Interestingly, progesterone and MPA both increased G-CSF and IL-8 secretion by ESCs, and these effects were apparently different from those of danazol (Fig. 4). These findings suggest that the effects of danazol on the 8-Br-cAMP-induced ESC activation process into G-CSF-secreting and IL-8-secreting cells are danazol-specific actions rather than progesterone-like actions.

\section{Discussion}

Local danazol therapy has been clinically applied to patients with endometriosis and adenomyosis because it attenuates their symptoms and reduces endometriotic and adenomyotic lesions without any severe adverse effects or significant serum endocrinological changes. Direct inhibition of endometrial cell proliferation has been believed to be the main mechanism of local danazol therapy. However, in vitro experiments using thoroughly dissolved danazol in $0.5 \%$ ethanol revealed that even high concentrations of danazol did not inhibit the proliferation rates of endometrial epithelial cells and ESCs (25). Moreover, we recently reported that danazol enhances Fas-mediated apoptosis within physiological variations (27). In the present study, we examined the effects of danazol on normal human ESCs. The present data further confirmed that danazol has no inhibitory effects on ESC growth, in contrast to previous studies $(24,26)$. Furthermore, this study is the first to identify a novel specific function by which danazol regulates the differentiation/activation process of functional ESC subpopulations.

The present experiments have revealed several unknown effects of danazol as described below. First, danazol did not affect ESC proliferation. Second, danazol did not affect the proliferation of 8-Br-cAMP-stimulated ESCs that are mainly composed of decidualized cells. Moreover, danazol did not affect PRL, G-CSF and IL-8 secretion by 8-Br-cAMPstimulated decidualized ESCs. Thus, no effects of danazol on 8-Br-cAMP-stimulated decidualized ESCs were detected in this study. Third, danazol dose-dependently enhanced PRL secretion by ESCs without 8-Br-cAMP stimulation, indicating that danazol has the ability to induce ESC decidualization. This decidualization-inducing activity of danazol may reflect a 

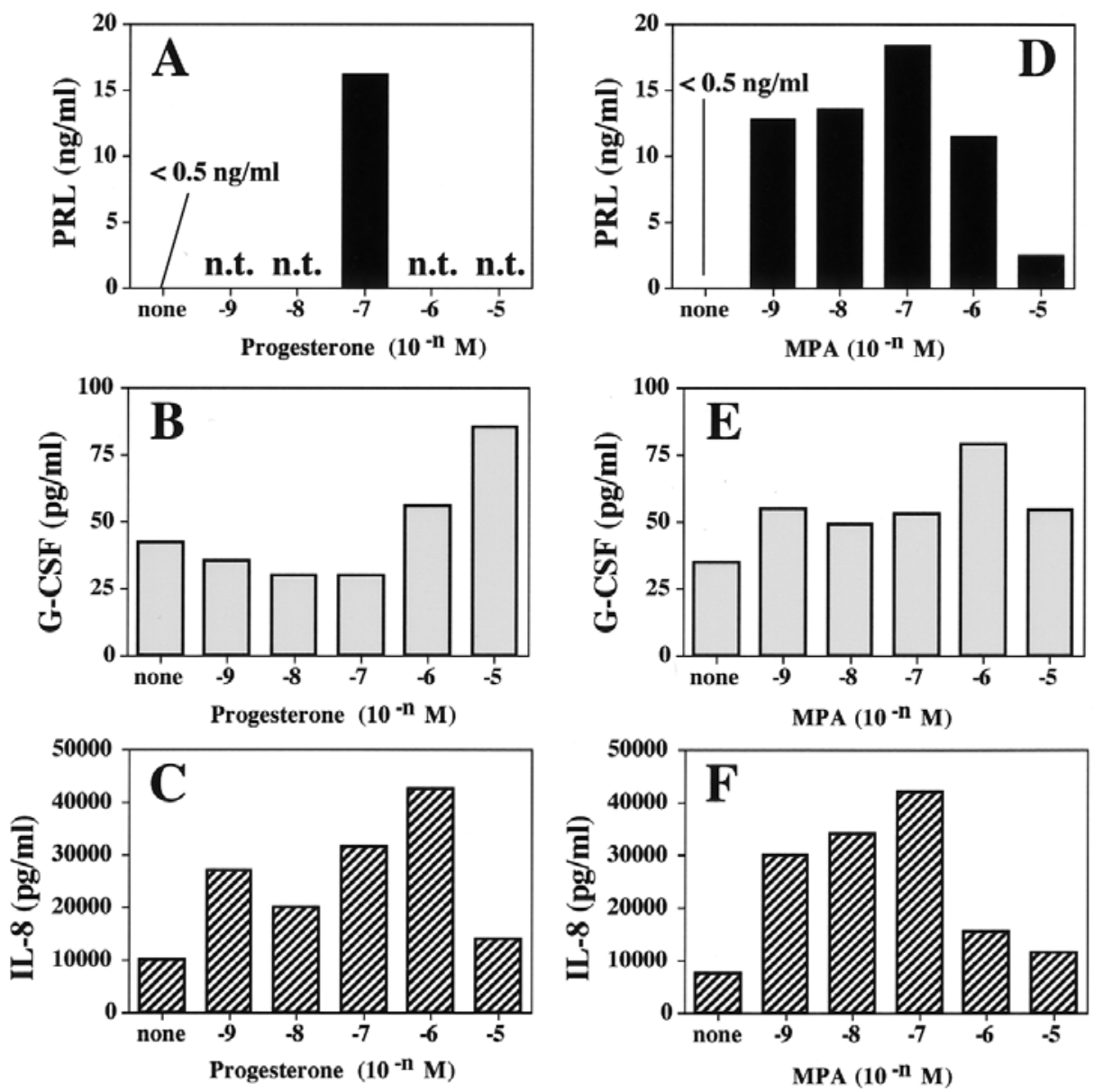

Figure 5. Effects of progesterone and MPA on PRL, G-CSF and IL-8 secretion by ESCs. (A-C) Effects of progesterone; (D-F) Effects of MPA; (A and D) PRL secretion; (B and E) G-CSF secretion; (C and F) IL-8 secretion; n.t., not tested. PRL secretion by unstimulated ESCs without progesterone or MPA treatment was $<0.5 \mathrm{ng} / \mathrm{ml}$ (in A and D). The data were presented as the mean values of the duplicated samples.

progesterone-like action. Fourth, danazol increased viable cell numbers of 8-Br-cAMP-stimulated ESCs during decidualization and increased PRL secretion by these cells. Since danazol did not increase PRL secretion by 8 -Br-cAMPstimulated decidualized ESCs, it is considered to synergistically enhance the decidualization process induced by 8 -Br-cAMP stimulation. This decidualization-enhancing activity of danazol may reflect a progesterone-like action of danazol, because progesterone synergistically enhances 8 -Br-cAMP-induced decidualization (29). Fifth, although danazol induced the differentiation of ESCs into PRL-secreting cells, i.e., decidualized cells, it did not affect G-CSF and IL-8 secretion by ESCs. On the other hand, progesterone and MPA induced decidualization of ESCs and increased G-CSF and IL-8 secretion by these cells. The latter effects differed from the effects of danazol on ESCs, indicating that danazol has specific pharmacological effects on ESCs rather than progesterone-like effects. Sixth, the activation process of ESCs into G-CSF- and IL-8-secreting cells was induced/enhanced by 8-Br-cAMP, progesterone and MPA, but not by danazol. Danazol was able to almost completely inhibit the 8-Br-cAMP-induced activation of ESCs into G-CSF- and IL-8-secreting cells, and it suppressed the levels of G-CSF and IL-8 secretion by ESCs to the basal levels observed without 8-Br-cAMP stimulation.
These findings suggest that the basal levels of G-CSF and IL-8 secretion by ESCs are not regulated by cAMP-related signals and that the regulatory system of these basal secretion levels is not affected by danazol and progesterone. Simultaneously, danazol strongly enhanced the 8-Br-cAMP-induced decidualization process. ESCs are hypothesized to consist of several subpopulations with different morphologies (32) and endocrinologically different functional subpopulations (30). PRL-secreting ESCs are hypothesized not to coincide with G-CSF-secreting ESCs (30). These results indicate that danazol regulates the functional cellular subpopulation ratios of normal human ESCs by mechanisms other than progesterone-like actions.

Limiting dilution analysis revealed that ESCs have several stable cellular subpopulations with morphologically different cell shapes (32). The responsiveness to macrophage colonystimulating factor (M-CSF) can be used to classify ESCs into at least the four subpopulations (30) as follows: ESC-1: unstimulated ESCs whose cell viability is not affected by MCSF, which secrete little PRL and G-CSF and whose G-CSF secretion but not PRL secretion can be enhanced by M-CSF; ESC-2: 8-Br-cAMP-stimulated G-CSF-producing ESCs whose cell viability and G-CSF secretion are not affected by M-CSF; ESC-3: 8-Br-cAMP-stimulated decidualizing cells 
whose cell viability is strongly suppressed by M-CSF; and ESC-4: 8-Br-cAMP-stimulated decidualized PRL-secreting cells whose cell viability and PRL secretion are not affected by M-CSF. By adding the present findings to the above-described classification, the four types of ESCs can be revised as follows: ESC-1: unstimulated ESCs whose cell viability is not affected by M-CSF and danazol, which secrete little PRL and G-CSF, whose G-CSF secretion but not PRL secretion can be enhanced by M-CSF and whose PRL secretion but not G-CSF/IL-8 secretion can be enhanced by danazol; ESC-2: 8-Br-cAMPstimulated G-CSF-producing ESCs whose cell viability and G-CSF secretion are not affected by M-CSF or danazol; ESC-3: 8-Br-cAMP-stimulated decidualizing cells whose cell viability is strongly suppressed by M-CSF and whose cell viability is strongly enhanced by danazol; and ESC-4: 8-BrcAMP-stimulated decidualized PRL-secreting cells whose cell viability and PRL secretion are not affected by M-CSF or danazol.

The mechanisms by which local danazol therapy improves the various signs and symptoms of endometriotic and adenomyotic patients without any severe adverse effects have remained unclear for a long time. Recently, we found that danazol enhances Fas-mediated apoptotic signals within physiological variations (27). The present study has revealed that danazol regulates the functional cellular subpopulation ratios of ESCs by different mechanisms from M-CSF (30). Danazol has a strong decidualization activity, similar to progesterone, but can strongly suppress the ESC activation process into G-CSF- and IL-8-secreting cells. The inhibitory effects of danazol on ESC activation into G-CSF-secreting cells are opposite to the effects of M-CSF, which induces ESC activation into G-CSF-secreting cells. M-CSF is produced in the human endometrium, particularly by decidual cells $(33,34)$. IL-8 secretion by ESCs is regulated by endometrial IL-1 and IL-1 receptor antagonist (31). Danazol enhanced the viability of 8-Br-cAMP-stimulated ESCs during decidualization, which is similar to the effects of several endometrial cytokines, including IL-11 (35), leukemia inhibitory factor (LIF) (36), oncostatin M (OSM) (37), leptin (38) and Fas ligand (FasL) (39). However, danazol did not affect the viability of 8-Br-cAMP-stimulated decidualized ESCs, which is different from the effects of IL-11, LIF, OSM, leptin and FasL (35-39). Therefore, under certain circumstances, danazol has similar or opposite effects on ESCs to certain endometrial cytokines, suggesting that it can modify the effects of the endometrial cytokine network in endometrial stromal tissues.

For patients with endometriosis and adenomyosis, gonadotropin-releasing hormone agonist therapy, which induces hypoestrogenemia and hypoprogesteronemia, is clinically effective. For these patients, oral contraceptive therapy composed of cyclic estrogen/progesterone therapy and progesterone therapy comprising a hyperprogesterone therapy are also clinically effective. These clinical facts suggest that the serum levels of estrogen and progesterone cannot be causes of endometriosis and adenomyosis, and that stable endocrinological environments can improve and reduce endometriosis and adenomyosis. Systemic endocrine imbalance must affect and disturb the cytokine networks in the endometrium. Danazol can modify these cytokine networks in the human endometrium and regulate the functional cellular subpopulation ratios of ESCs, and these effects may reflect the main mechanisms of actions of safe local danazol therapy for endometriosis and adenomyosis.

\section{Acknowledgements}

This study was supported in part by a Grant-in-Aid for Scientific Research from the Ministry of Education, Science, Sports and Culture of Japan. We would like to thank TokyoTanabe Pharmaceutical Co. Ltd. for donating the danazol.

\section{References}

1. Wentz AC, Jones GS, Sapp KC and King TM: Progestational activity of danazol in the human female subject. Am J Obstet Gynecol 126: 378-384, 1976.

2. Kokko E, Jaenne O, Kauppila A, Roennberg L and Vihko R: Danazol has progesterone-like actions on the human endometrium. Acta Endocrinol 99: 588-593, 1982.

3. Potts GO, Beyler AL and Schane HP: Pituitary gonadotropin inhibitory activity of danazol. Fertil Steril 25: 367-372, 1974.

4. Wood GP: hormonal changes associated with danazol therapy. Obstet Gynecol 45: 302-304, 1975.

5. Andrews MC and Wentz AC: The effects of danazol on gonadotropins and steroid blood levels in normal and anovulatory women. Am J Obstet Gynecol 121: 817-828, 1975.

6. Barbieri RL: Danazol inhibits steroidgenesis. Fertil Steril 28: 809-813, 1977.

7. Dmowski WP: Endocrine properties and clinical application of danazol. Fertil Steril 31: 237-251, 1979.

8. Hirshowitz JS, Soler NG and Wortsman J: Sex steroid levels during treatment of endometriosis. Obstet Gynecol 54: 448-450, 1979.

9. Floyd WS: Danazol: Endocrine and endometrial effects. Int J Fertil 25: 75-80, 1980.

10. Barbieri RL: Danazol inhibition of steroidgenesis in the human corpus luteum. Obstet Gynecol 57: 722-724, 1981.

11. Barbieri RL and Ryan KJ: Danazol: Endocrine pharmacology and therapeutic applications. Am J Obstet Gynecol 141: 453-463, 1981.

12. Meldrum DR, Pardridge WM, Karow WG, Rivier J, Vale W and Judd HL: Hormonal effects of danazol and medical oophorectomy in endometriosis. Obstet Gynecol 62: 480-485, 1983

13. Igarashi M: A new therapy for pelvic endometriosis and uterine adenomyosis: local effect of vaginal and intrauterine Danazol application. Asia-Oceania J Obstet Gynecol 16: 1-12, 1990.

14. Takeda Y, Yano J, Hukui K, Kitagawa H, Matsuura S, Yano H and Yano C: Danazol concentration in peritoneal fluid: A comparative study of oral danazol capsule therapy and vaginal danazol suppository therapy. J Jpn Soc Endometriosis 14: 216-221, 1993.

15. Samejima T, Ishimaru T, Masuzaki H, Hujishita N, Nakamura K, Matsuwaki $\mathrm{T}$ and Yamabe T: Effects of danazol vaginal suppository onendometriosis. J Jpn Soc Endometriosis 14: 222-225, 1993.

16. Ito K, Itani Y, Kurai N, Tamori C, Noda T, Katoh Y, Hisanaga H, Sintani M, Kawai S, Beppu K, Saitou K, Adachi S and Nakayama M: Danazol vaginal tablet: clinical effects and tissue concentration of danazol. J Jpn Soc Endometriosis 15: 202-204, 1994.

17. Takebayashi T, Fujino Y, Umesaki N and Ogita S: Danazol suspension injected into the uterine cervix of patients with adenomyosis and myoma. Preliminary study. Gynecol Obstet Invest 39: 207-211, 1995.

18. Igarashi M, Iizuka M, Abe Y and Ibuki Y: Novel vaginal danazol ring therapy for pelvic endometriosis, in particular deeply infiltrating endometriosis. Hum Reprod 13: 1952-1956, 1998

19. Igarashi M, Abe Y, Fukuda M, Ando A, Miyasaka M, Yoshida M and Shawki OA: Novel conservative medical therapy for uterine adenomyosis with a danazol-loaded intrauterine device. Fertil Steril 74: 412-413, 2000

20. Terakawa N, Ikegami H, Shimizu I, Aono T, Tanizawa O and Matsumoto K: Growth inhibition by Danazol in a human endometrial cancer cell line with estrogen-independent progesterone receptors. J Steroid Biochem 28: 571-574, 1987. 
21. Terakawa N, Ikegami H, Shimizu I, Inoue M, Tanizawa $\mathrm{O}$ and Matsumoto K: Inhibitory effects of Danazol and medroxyprogesterone acetate on $[3 \mathrm{H}]$ thymidine incorporation in human endometrial cancer cells. J Steroid Biochem 31: 131-135, 1988.

22. Taketani Y and Mizuno M: Direct actions of danazol on endometrium. Obstet Gynecol 52: 89-91, 1985.

23. Rose GL, Dowsett M, Mudge JE, White JO and Jeffcoate SL: The inhibitory effects of danazol, danazol metabolites, gestrinone, and testosterone on the growth of human endometrial cells in vitro. Fertil Steril 49: 224-228, 1988.

24. Surrey ES and Halme J: Direct effects of medroxyprogesterone acetate, danazol, and leuprolide acetate on endometrial stromal cell proliferation in vitro. Fertil Steril 58: 273-278, 1992.

25. Tanaka T, Umesaki N, Chen H, Mizuno K and Ogita S: Danazol effects on human endometrial cells in vitro. Clin Exp Obstet Gynecol 26: 67-70, 1999.

26. Taguchi M, Kubota T and Aso T: Direct effect of danazol on the DNA synthesis and ultrastructure of human cultured endometrial stromal cells. Gynecol Obstet Invest 39: 192-196, 1995.

27. Tanaka $T$ and Umesaki N: Danazol enhances Fas-mediated apoptosis in human endometrial epithelial cells within normal physiology. Int J Mol Med 23: 237-243, 2009.

28. Tanaka T, Masuda M, Mizuno K, Sakamoto T, Miyama M, Umesaki $\mathrm{N}$ and Ogita $\mathrm{S}$ : In vitro decidualization activity assay of human endometrial stromal cells. Clin Exp Obstet Gynecol 26: 166-170, 1999.

29. Mizuno K, Tanaka T, Umesaki N and Ogita S: Establishment and characterization of in vitro decidualization in normal human endometrial stromal cells. Osaka City Med J 44: 105-115, 1998.

30. Tanaka $\mathrm{T}$ and Umesaki N: Regulation of the cellular subpopulation ratios of normal human endometrial stromal cells by macrophage colony-stimulating factor. Int $\mathbf{J}$ Mol Med 11: $617-620,2003$
31. Tanaka T, Sakamoto T, Mizuno K, Umesaki N and Ogita S: Human endometrial stromal interleukin-1ß: Autocrine secretion and inhibition by interleukin-1 receptor antagonist. Hormone Res 53: 300-304, 2000.

32. Tanaka T, Nakajima S and Umesaki N: Cellular heterogeneity in long-term surviving cells isolated from eutopic endometrial, ovarian endometrioma and adenomyosis tissues. Oncol Rep 10: 1150-1160, 2003

33. Kariya M, Kanzaki H, Hanamura T, Imai K, Narukawa S, Inoue $\mathrm{T}$, Hatayama $\mathrm{H}$ and Mori T: Progesterone-dependent secretion of macrophage colony-stimulating factor by human endometrial stromal cells of nonpregnant uterus in culture. J Clin Endocrinol Metab 79: 86-90, 1994

34. Hatayama H, Kanzaki H, Iwai M, Kariya M, Fujimoto M, Higuchi T, Kojima K, Nakayama H, Mori T and Fujita J: Progesterone enhances macrophage colony-stimulating factor production in human endometrial stromal cells in vitro. Endocrinology 135: 1921-1927, 1994.

35. Tanaka T, Sakamoto T, Umesaki N and Ogita S: Interleukin-11 enhances cell survival of decidualized normal human endometrial stromal cells. Gynecol Endocrinol 15: 272-278, 2001.

36. Nakajima S, Tanaka T, Umesaki N and Ishiko O: Leukemia inhibitory factor regulates cell survival of normal human endometrial stromal cells. Int J Mol Med 11: 353-356, 2003

37. Tanaka T and Umesaki N: Oncostatin M inhibits decidualization of normal human endometrial stromal cells. Int J Mol Med 11: 627-630, 2003.

38. Tanaka T, Utsunomiya T, Bai T, Nakajima S and Umesaki N: Leptin inhibits decidualization and enhances cell viability of normal human endometrial stromal cells. Int J Mol Med 12: 95-98, 2003.

39. Tanaka $\mathrm{T}$ and Umesaki N: Fas antigen (CD95) mediates cell survival signals to regulate functional cellular subpopulations in normal human endometrial stromal cells. Int J Mol Med 11: 757-762, 2003. 